\author{
Series A
}

\author{
I. MATHEMATICA
}

335

\title{
SOME APPROXIMATION THEOREMS FOR NORMAL FUNCTIONS
}

FREDERICK BAGEMIHL 
Communicated 9 April 1963 by P. J. Myrberg and Olli Lehto 


\section{Some approximation theorems for normal functions ${ }^{1}$ )}

1. Suppose that $f(z)$ is a nonconstant normal meromorphic function (see $[2, \mathrm{p} .86]$ ) in the open unit disk $D$. We are going to show, among other things, that if the set $A(f)$ of asymptotic values of $f$ is of harmonic measure zero, then there exists a set $S$ of points on the unit circle $T$, where $S$ is both of Lebesgue measure $2 \pi$ and a residual set in the sense of Baire category, with the following property: the function $f(z)$ approximates every complex value arbitrarily closely on every continuous curve in $D$ that intersects $\Gamma$ in a point of $S$ at an angle different from zero.

This generalizes in several directions certain classical results of $P$. J. Myrberg ([3], [4]). (A very special case to which our theorem applies is that of the elliptic modular function.)

2. We denote by $\Omega$ the extended complex plane. If $z$ and $z^{\prime}$ are points of $D$, then $\varrho\left(z, z^{\prime}\right)$ is the non-Euclidean hyperbolic distance between them. A Stolz angle with vertex $\zeta \in \Gamma$ will be called a Stolz angle at $\zeta$. By an arc at $\zeta \in \Gamma$ we mean a continuous curve $\Lambda: z=z(t)$ $(0 \leqq t<1)$ such that $|z(t)|<1$ for $0 \leqq t<1$ and $\lim _{t \rightarrow 1} z(t)=\zeta$. A terminal subarc of an arc $\Lambda$ at $\zeta$ is a subarc of $\Lambda$ of the form $z=z(t)$ $\left(t_{0} \leqq t<1\right)$, where $0 \leqq t_{0}<1$. By an admissible arc at $\zeta$ we mean an arc at $\zeta$ having a tangent at $\zeta$ different from the tangent to $\Gamma$ at $\zeta$.

If $f(z)$ is meromorphic in $D$, and $\zeta \in \Gamma$, the cluster set of $f$ at $\zeta$ is denoted by $C(f, \zeta)$; the cluster set of $f$ at $\zeta$ on an arc $A$ at $\zeta$, or on a Stolz angle $\Delta$ at $\zeta$, is denoted by $C_{\perp}(f, \zeta), C_{\Delta}(f, \zeta)$, respectively. We write $C_{\mathscr{\gamma}}(f, \zeta)=\bigcup_{\Delta} C_{\Delta}(f, \zeta)$, where the union is taken over all Stolz angles $\Delta$ at $\zeta$. We define $\Pi_{T}(f, \zeta)=\bigcap_{A} C_{A}(f, \zeta)$, where the intersection

1) Presented at the Conference on the Theory of Functions of a Single Complex Variable, Mathematisches Forschungsinstitut, Oberwolfach, Germany, March 25, 1963. 
is taken over all admissible $\operatorname{arcs} A$ at $\zeta$. The set of points $\zeta \in \Gamma$ such that $C_{\Delta}(f, \zeta)=C_{\Delta^{\prime}}(f, \zeta)$ for every pair of Stolz angles $\Delta, \Delta^{\prime}$ at $\zeta$ is called $K(f)$. (The set $K(f)$ is always a residual subset of $\Gamma$ of measure $2 \pi[2$, p. 68].)

3. Our theorems are based upon the following general

Lemma. Let $f(z)$ be a normal meromorphic function in $D$, and suppose that $\zeta \in K(f)$. Then $\Pi_{T}(f, \zeta)=C_{\mathcal{X}}(f, \zeta)$.

Proof: Suppose that $\omega \in \Pi_{T}(f, \zeta)$. Then $\omega \in C_{A}(f, \zeta)$ for every admissible arc $\Lambda$ at $\zeta$. Since $\Lambda$ intersects $\Gamma$ at a nonzero angle, there exists a Stolz angle $\Delta$ at $\zeta$ containing a terminal subarc of $\Lambda$. Clearly $C_{A}(f, \zeta) \subseteq C_{\Delta}(f, \zeta)$, so that $\omega \in C_{\mathscr{\gamma}}(f, \zeta)$.

Now suppose that $\omega \in C_{\mathcal{X}}(f, \zeta)$. Let $\Lambda$ be any admissible arc at $\zeta$. Since $\zeta \in K(f)$, we have $\omega \in C_{\Delta}(f, \zeta)$ for every Stolz angle $\Delta$ at $\zeta$, and hence there exists a sequence of points $\left\{z_{n}^{\prime}\right\}$ in $D$, where $\lim z_{n}^{\prime}=\zeta$ and $\lim _{n \rightarrow \infty} f\left(z_{n}^{\prime}\right)=\omega$, such that, for an appropriate sequence of points $\left\{z_{n}\right\}$ on $\Lambda$ with $\lim _{n \rightarrow \infty} z_{n}=\zeta$, we have $\lim _{n \rightarrow \infty} \varrho\left(z_{n}, z_{n}^{\prime}\right)=0$. From the fact that $f(z)$ is a normal meromorphic function in $D$, we infer $[1$, p. 10, Lemma 1] that $\lim _{n \rightarrow \infty} f\left(z_{n}\right)=\omega$, and hence $\omega \in C_{A}(f, \zeta)$. This holds for an arbitrary admissible arc $\Lambda$ at $\zeta$, and therefore $\omega \in \Pi_{T}(f, \zeta)$.

4. Theorem 1. Let $f(z)$ be a nonconstant normal meromorphic function in $D$, and suppose that $A(f)$ is of harmonic measure zero. Then there exists a residual subset $S$ of $\Gamma$ of measure $2 \pi$ such that, for every $\zeta \in S$, $\Pi_{T}(f, \zeta)=\Omega$.

Proof: According to Plessner's theorem [2, p. 70], almost every point of $\Gamma$ is either a Fatou point or a Plessner point of $f$. The set of Fatou points of $f$, however, must be of measure zero, otherwise Privalov's theorem [2, p. 72] would imply a contradiction to the fact that $f$ is nonconstant and $A(f)$ is of harmonic measure zero. Hence, the set $I(f)$ of Plessner points of $f$ is of measure $2 \pi$. It follows $[2$, p. 65] that $I(f)$ is also a residual subset of $\Gamma$. If $\zeta \in I(f)$, then $C_{\not \gamma}(f, \zeta)=\Omega$, and since $I(f) \subset K(f)$, our Lemma yields $\Pi_{T}(f, \zeta)=C_{\not \gamma}(f, \zeta)$. Setting $S=I(f)$, we obtain Theorem 1 .

A set of linear measure zero may be of positive harmonic measure $[2$, p. 7]. In case $A(f)$ is known merely to be of linear measure zero, we have nevertheless the following result.

Theorem 2. Let $f(z)$ be a nonconstant normal meromorphic function in $D$, and suppose that $A(f)$ is of linear measure zero. Then there exists a residual subset $R$ of $\Gamma$ such that, for every $\zeta \in R, \Pi_{T}(f, \zeta)=\Omega$. 
Proof: Since $f(z)$ is nonconstant and $A(f)$ is of linear measure zero, we have $C(f, \zeta)=\Omega$ for every $\zeta \in \Gamma[2$, p. 51]. It follows [2, III, $\S 3]$ that $I(f)$ is a residual subset of $\Gamma$. As in the proof of Theorem $1, \zeta \in I(f)$ implies that $\Pi_{T}(f, \zeta)=\Omega$, and setting $R=I(f)$, we obtain Theorem 2 .

Wayne State University

Detroit, Michigan, U.S. A.

\section{References}

[1] F. Bagemihl and W. Seidel, Sequential and continuous limits of meromorphic functions.- Ann. Acad. Sci. Fennicæ A I 280 (1960), $1-17$.

[2] K. Noshiro, Cluster sets.- Berlin, 1960.

[3] P. J. Myrberg, Ein Satz über die fuchsschen Gruppen und seine Anwendung in der Funktionentheorie.- Ann. Acad. Sci. Fennicæ A 32 (1929) No. 9, $1-35$.

[4] -»- Ein Approximationssatz für die fuchsschen Gruppen.- Acta Math. 57 (1931), 389-409. 